\title{
AN ELLIPTIC PROBLEM WITH TWO SINGULARITIES
}

\author{
GISELLA CROCE
}

\begin{abstract}
We study a Dirichlet problem for an elliptic equation defined by a degenerate coercive operator and a singular right-hand side. We will show that the right-hand side has some regularizing effects on the solutions, even if it is singular.
\end{abstract}

\section{INTRODUCTION}

In this note we study existence of solutions to the following elliptic problem:

$$
\left\{\begin{array}{cl}
-\operatorname{div}\left(\frac{a(x) \nabla u}{(1+|u|)^{p}}\right)=\frac{f}{|u|^{\gamma}} & \text { in } \Omega, \\
u=0 & \text { on } \partial \Omega,
\end{array}\right.
$$

where $\Omega$ is an open bounded subset of $\mathbb{R}^{N}, N \geq 3, p$ and $\gamma$ are positive reals, $f$ is an $L^{m}(\Omega)$ non-negative function and $a: \Omega \rightarrow \mathbb{R}$ is a measurable function such that $0<\alpha \leq a(x) \leq \beta$, for two positive constants $\alpha$ and $\beta$.

The operator $v \rightarrow-\operatorname{div}\left(\frac{a(x) \nabla v}{(1+|v|)^{p}}\right)$ is not coercive on $H_{0}^{1}(\Omega)$, when $v$ is large (see [13]); moreover, the right-hand side is singular in the variable $u$. These two difficulties has led us to study problem (1.1) by approximation. More precisely, we will define a sequence of problems (see problems (2.1)), depending on the parameter $n \in \mathbb{N}$, in which we "truncate" the degenerate coercivity of the operator term and the singularity of the right hand side. We will prove in Section 2 that these problems admit a bounded $H_{0}^{1}(\Omega)$ solution $u_{n}, n \in \mathbb{N}$, with the property that for every subset $\omega \subset \subset \Omega$ there exists a positive constant $c_{\omega}>0$ such that $u_{n} \geq c_{\omega}$ almost everywhere in $\omega$ for every $n \in \mathbb{N}$. In Section 3 we will get some a priori estimates on $u_{n}$ and, by compactness, a function $u$ to which $u_{n}$ converges. By passing to the limit in the approximating problems (2.1), $u$ will turn out to be a solution to problem (1.1) in the following sense. For every $\omega \subset \subset \Omega$ there exists $c_{\omega}>0$ such that $u \geq c_{\omega}>0$ in $\omega$ and

$$
\int_{\Omega} a(x) \frac{\nabla u \cdot \nabla \varphi}{(1+u)^{p}}=\int_{\Omega} \frac{f}{u^{\gamma}} \varphi \quad \forall \varphi \in C_{0}^{1}(\Omega) .
$$

We are now going to motivate the study of problem (1.1). The lack of coercivity of the operator term can negatively affect the existence and regularity of solutions to

$$
\left\{\begin{array}{cl}
-\operatorname{div}\left(\frac{a(x) \nabla u}{(1+|u|)^{p}}\right)=f & \text { in } \Omega, \\
u=0 & \text { on } \partial \Omega .
\end{array}\right.
$$

This was first pointed out in [7, in the case $p<1$. In the case where $p>1$, the authors of [1] proved that no solutions exist for some constant sources $f$.

A natural question is then to search for lower order terms which regularize the solutions to problem (1.3). This leads to the study of existence and regularity of solutions to problems 
of the form

$$
\left\{\begin{array}{cl}
-\operatorname{div}\left(\frac{a(x) \nabla u}{(1+|u|)^{p}}\right)+g(u, \nabla u)=f & \text { in } \Omega, \\
u=0 & \text { on } \partial \Omega,
\end{array}\right.
$$

under various hypotheses on $g: \Omega \times \mathbb{R}^{N} \rightarrow \mathbb{R}$. In [6] the case $g=g(s)=s$ was examined. This work takes advantage of the fact that, just as for semilinear elliptic coercive problems (cf. 9), the summability of the solutions is at least that of the source $f$. In [10] we analysed two different lower order terms $g=g(s)$. The first, a generalization of the lower order term studied in [6], is $g(s)=|s|^{r-1} s, r>0$. The second is a continuous positive increasing function such that $g(s) \rightarrow+\infty$, as $s \rightarrow s_{0}^{-}$, for some $s_{0}>0$ (see [3]). The lower order term of [4] is, roughly speaking, $b(|s|)|\xi|^{2}$ where $b$ is continuous and increasing with respect to $|s|$. In [11] we showed the regularizing effects of the lower order term $g(s, \xi)=\frac{|\xi|^{2}}{s^{q}}, q>0$, which grows as a negative power with respect to $s$ and has a quadratic dependence on the gradient variable (see [2] and [5] for elliptic coercive problems with the same lower order term).

In all of the above papers a regularizing effect on the solutions to (1.3) was shown under a sign condition on $g$ : either $g(s, \xi) \geq 0$ for every $(s, \xi) \in \mathbb{R} \times \mathbb{R}^{N}$ and the source is assumed to be positive or $g(s, \xi) s \geq 0$ for every $(s, \xi) \in \mathbb{R} \times \mathbb{R}^{N}$.

In this paper we study the effects on the solutions of a different term, $\frac{f}{u^{\gamma}}$, on the right hand side. Our inspiration is taken from 8 where the authors considered the same right hand side, for elliptic semilinear problems whose model is $-\Delta u=\frac{f}{u^{\gamma}}$, with zero Dirichlet condition on the boundary. Our main result shows that, despite the singularity in $u$, this term has some regularizing effects on the solutions to (1.3). The regularity depends on the different values of $\gamma-p$ : we distinguish the cases $p-1 \leq \gamma<p+1, \gamma=p+1$, and $\gamma>p+1$. These statements are made more precise in the following

TheOREm 1.1. Let $\gamma \geq p-1$.

(1) Let $\gamma<p+1$.

(a) If $f \in L^{m}(\Omega)$, with $m \geq \frac{2^{*}}{2^{*}-p-1+\gamma}$, there exists a solution $u \in H_{0}^{1}(\Omega)$ to (1.1) in the sense of (1.2). If $\frac{2^{*}}{2^{*}-p-1+\gamma} \leq m<\frac{N}{2}$, then $u$ belongs to $L^{m^{* *}(\gamma+1-p)}(\Omega)$.

(b) If $f \in L^{m}(\Omega)$, with $\max \left\{1, \frac{1^{*}}{2 \cdot 1^{*}-p-1+\gamma}\right\}<m<\frac{2^{*}}{2^{*}-p-1+\gamma}$, there exists a solution $u \in W_{0}^{1, \sigma}(\Omega), \sigma=\frac{N m(\gamma+1-p)}{N-m(p+1-\gamma)}$, to (1.1) in the sense of (1.2).

(2) Let $\gamma=p+1$ and assume that $f \in L^{1}(\Omega)$. Then there exists a solution $u \in H_{0}^{1}(\Omega)$ to (1.1) in the sense of (1.2).

(3) Let $\gamma>p+1$ and assume that $f \in L^{1}(\Omega)$. Then there exists a solution $u \in H_{l o c}^{1}(\Omega)$ to (1.1) in the sense of (1.2), such that $u^{\frac{\gamma+1-p}{2}} \in H_{0}^{1}(\Omega)$.

(4) Let $f \in L^{m}(\Omega)$, with $m>\frac{N}{2}$. Then the solution found above is bounded. 
Let us point out the regularizing effects of the right hand side. It is useful to recall the results obtained in [7] for problem (1.3). Let $p<1$ and $q=\frac{N m(1-p)}{N-m(1+p)}$.

a) If $1<m \leq \frac{2 N}{N+2-p(N-2)}$, then there exists $u \in W_{0}^{1, q}(\Omega)$ or $|\nabla u|^{s} \in L^{1}(\Omega)$, $\forall s<q$.

b) If $\frac{2 N}{N+2-p(N-2)} \leq m<\frac{N}{2}$, then there exists $u \in H_{0}^{1}(\Omega) \cap L^{m^{* *}(1-p)}(\Omega)$.

c) If $m>\frac{N}{2}$, then there exists $u \in H_{0}^{1}(\Omega) \cap L^{\infty}(\Omega)$.

We now compare the summabilities obtained in Theorem 1.1 to the previous ones. First of all, we have a solution for every $p>0$, if $\gamma \geq p-1$. This is not the case for problem (1.3), as proved in [1. Under the same conditions on $f$, the summability of the solutions to (1.1) is better than or equal to that of the solutions to (1.3), since $\sigma>q$ and $m^{* *}(\gamma+1-p)>$ $m^{* *}(1-p)$. Moreover, we get $H_{0}^{1}(\Omega)$ solutions for less regular sources than in [7]. Indeed, if $p-1 \leq \gamma<p+1$, one has $\frac{2^{*}}{2^{*}-p-1+\gamma}<\frac{2 N}{N(1-p)+2(p+1)}$; if $\gamma=p+1$ we get a finite energy solution for every $L^{1}(\Omega)$ source.

\section{Approximating problems}

As explained in the Introduction, we will work on the following approximating problems:

$$
\left\{\begin{aligned}
-\operatorname{div}\left(\frac{a(x) \nabla u_{n}}{\left(1+\left|T_{n}\left(u_{n}\right)\right|\right)^{p}}\right)=\frac{T_{n}(f)}{\left(\left|u_{n}\right|+\frac{1}{n}\right)^{\gamma}} & \text { in } \Omega, \\
u_{n}=0 & \text { on } \partial \Omega
\end{aligned}\right.
$$

where $n \in \mathbb{N}$ and

$$
T_{n}(s)= \begin{cases}-n, & s \leq-n \\ s, & -n \leq s \leq n \\ n, & s \geq n .\end{cases}
$$

Observe that we have "truncated" the degenerate coercivity of the operator term and the singularity of the right hand side.

Proposition 2.1. Problems (2.1) are well posed, that is, there exists a non-negative solution $u_{n} \in H_{0}^{1}(\Omega) \cap L^{\infty}(\Omega)$ for every fixed $n \in \mathbb{N}$.

Proof. In this proof we will use the same technique as in 8 . Let $S: L^{2}(\Omega) \rightarrow L^{2}(\Omega)$ be the map which associates to every $v \in L^{2}(\Omega)$ the solution $w_{n} \in H_{0}^{1}(\Omega)$ to

$$
\left\{\begin{array}{cc}
-\operatorname{div}\left(\frac{a(x) \nabla w_{n}}{\left(1+\left|T_{n}\left(w_{n}\right)\right|\right)^{p}}\right)=\frac{T_{n}(f)}{\left(|v|+\frac{1}{n}\right)^{\gamma}} & \text { in } \Omega, \\
w_{n}=0 & \text { on } \partial \Omega .
\end{array}\right.
$$

Observe that $S$ is well-defined by the results of 12 and $w_{n}$ is bounded by the results of [14. Let us choose $w_{n}$ as a test function. Then,

$$
\alpha \int_{\Omega} \frac{\left|\nabla w_{n}\right|^{2}}{(1+n)^{p}} \leq \int_{\Omega} \frac{a(x)\left|\nabla w_{n}\right|^{2}}{\left(1+T_{n}\left(w_{n}\right)\right)^{p}}=\int_{\Omega} \frac{T_{n}(f) w_{n}}{\left(|v|+\frac{1}{n}\right)^{\gamma}} \leq n^{\gamma+1} \int_{\Omega}\left|w_{n}\right| \leq|\Omega|^{1 / 2} n^{\gamma+1}\left\|w_{n}\right\|_{L^{2}(\Omega)}
$$


by the hypotheses on $a$ and Hölder's inequality on the right hand side. Poincaré's inequality on the left hand side implies

$$
\alpha \mathcal{P}\left\|w_{n}\right\|_{L^{2}(\Omega)}^{2} \leq|\Omega|^{1 / 2}(1+n)^{p} n^{\gamma+1}\left\|w_{n}\right\|_{L^{2}(\Omega)} .
$$

Thus there exists an invariant ball for $S$. Moreover it is easily seen that $S$ is continuous and compact by the $H_{0}^{1}(\Omega) \hookrightarrow L^{2}(\Omega)$ embedding. By Schauder's theorem, $S$ has a fixed point. Therefore there exists a solution $u_{n} \in H_{0}^{1}(\Omega)$ to problems (2.1). Observe that $u_{n}$ is bounded; by the maximum principle, $u_{n}$ is non-negative since $f$ is non-negative.

REMARK 2.2. We remark that the existence of solutions to (2.1) could not be inferred by the results established in [7].

Proposition 2.3. Let $u_{n}$ be the solution to problem [2.1). Then $u_{n} \leq u_{n+1}$ a.e. in $\Omega$. Moreover for every $\omega \subset \subset \Omega$ there exists $c_{\omega}>0$ such that $u_{n} \geq c_{\omega}$ a.e. in $\omega$ for every $n \in \mathbb{N}$.

Proof. We will use the same technique as in 13 to prove uniqueness of the solutions to problem (1.3). In the proof $C$ will denote a positive constant independent of $n$ (depending on $\alpha, \beta, p$ and the constant $\mathcal{P}$ of Poincaré's inequality). The solution $u_{n}$ to problem (2.1) satisfies

$$
-\operatorname{div}\left(\frac{a(x) \nabla u_{n}}{\left(1+T_{n}\left(u_{n}\right)\right)^{p}}\right)=\frac{T_{n}(f)}{\left(u_{n}+\frac{1}{n}\right)^{\gamma}} \leq \frac{T_{n+1}(f)}{\left(u_{n}+\frac{1}{n+1}\right)^{\gamma}} .
$$

Therefore

$$
-\operatorname{div}\left(\frac{a(x) \nabla u_{n}}{\left(1+T_{n}\left(u_{n}\right)\right)^{p}}-\frac{a(x) \nabla u_{n+1}}{\left(1+T_{n+1}\left(u_{n+1}\right)\right)^{p}}\right)=T_{n+1}(f)\left[\frac{1}{\left(u_{n}+\frac{1}{n+1}\right)^{\gamma}}-\frac{1}{\left(u_{n+1}+\frac{1}{n+1}\right)^{\gamma}}\right] \leq 0 .
$$

By choosing $T_{k}\left(\left(u_{n}-u_{n+1}\right)^{+}\right)$as a test function we get

$$
\begin{gathered}
\alpha \int_{\Omega} \frac{\left|\nabla T_{k}\left(\left(u_{n}-u_{n+1}\right)^{+}\right)\right|^{2}}{\left(1+T_{n}\left(u_{n}\right)\right)^{p}} \\
\leq \beta \int_{\Omega} \nabla u_{n+1} \cdot \nabla T_{k}\left(\left(u_{n}-u_{n+1}\right)^{+}\right)\left[\frac{1}{\left(1+T_{n+1}\left(u_{n+1}\right)\right)^{p}}-\frac{1}{\left(1+T_{n}\left(u_{n}\right)\right)^{p}}\right]
\end{gathered}
$$

by the hypotheses on $a$. In $\left\{0 \leq u_{n}-u_{n+1} \leq k\right\}$ one has

$$
\left|\frac{1}{\left(1+T_{n+1}\left(u_{n+1}\right)\right)^{p}}-\frac{1}{\left(1+T_{n}\left(u_{n}\right)\right)^{p}}\right| \leq k\left[\frac{1}{\left(1+T_{n+1}\left(u_{n+1}\right)\right)^{p}}+\frac{1}{\left(1+T_{n}\left(u_{n}\right)\right)^{p}}\right] .
$$

Therefore

$$
\begin{gathered}
\int_{\Omega} \frac{\left|\nabla T_{k}\left(\left(u_{n}-u_{n+1}\right)^{+}\right)\right|^{2}}{\left(1+T_{n}\left(u_{n}\right)\right)^{p}} \leq \\
\int_{\left\{0 \leq u_{n}-u_{n+1} \leq k\right\}}\left|\nabla u_{n+1}\right|\left|\nabla T_{k}\left(\left(u_{n}-u_{n+1}\right)^{+}\right)\right|\left|\frac{1}{\left(1+T_{n+1}\left(u_{n+1}\right)\right)^{p}}+\frac{1}{\left(1+T_{n}\left(u_{n}\right)\right)^{p}}\right| .
\end{gathered}
$$

For sufficiently small $k$, one has in $\left\{0 \leq u_{n}-u_{n+1} \leq k\right\}$

$$
\frac{1}{2^{p}} \frac{1}{\left(1+T_{n}\left(u_{n}\right)\right)^{p}} \leq \frac{1}{\left(1+T_{n+1}\left(u_{n+1}\right)\right)^{p}} \leq \frac{2^{p}}{\left(1+T_{n}\left(u_{n}\right)\right)^{p}} .
$$

This implies that

$$
\int_{\Omega} \frac{\left|\nabla T_{k}\left(\left(u_{n}-u_{n+1}\right)^{+}\right)\right|^{2}}{\left(1+T_{n}\left(u_{n}\right)\right)^{p}} \leq C k \int_{\left\{0 \leq u_{n}-u_{n+1} \leq k\right\}} \frac{\left|\nabla u_{n+1}\right|}{\left(1+T_{n+1}\left(u_{n+1}\right)\right)^{p / 2}} \frac{\left|\nabla T_{k}\left(\left(u_{n}-u_{n+1}\right)^{+}\right)\right|}{\left(1+T_{n}\left(u_{n}\right)\right)^{p / 2}} .
$$


Hölder's inequality on the right hand side gives

$$
\begin{gathered}
\int_{\Omega} \frac{\left|\nabla T_{k}\left(\left(u_{n}-u_{n+1}\right)^{+}\right)\right|^{2}}{\left(1+T_{n}\left(u_{n}\right)\right)^{p}} \\
\leq C k\left[\int_{\left\{0 \leq u_{n}-u_{n+1} \leq k\right\}} \frac{\left|\nabla u_{n+1}\right|^{2}}{\left(1+T_{n+1}\left(u_{n+1}\right)\right)^{p}}\right]^{\frac{1}{2}}\left[\int_{\left\{0 \leq u_{n}-u_{n+1} \leq k\right\}} \frac{\left|\nabla T_{k}\left(\left(u_{n}-u_{n+1}\right)^{+}\right)\right|^{2}}{\left(1+T_{n}\left(u_{n}\right)\right)^{p}}\right]^{\frac{1}{2}},
\end{gathered}
$$

and then

$$
\int_{\Omega} \frac{\left|\nabla T_{k}\left(\left(u_{n}-u_{n+1}\right)^{+}\right)\right|^{2}}{\left(1+T_{n}\left(u_{n}\right)\right)^{p}} \leq C k^{2} \int_{\left\{0 \leq u_{n}-u_{n+1} \leq k\right\}} \frac{\left|\nabla u_{n+1}\right|^{2}}{\left(1+T_{n+1}\left(u_{n+1}\right)\right)^{p}} .
$$

On the other hand, by Poincaré's inequality and (2.4)

that is,

$$
\begin{gathered}
k^{2}\left|\left\{0 \leq u_{n}-u_{n+1} \leq k\right\}\right| \leq \int_{\Omega} \frac{\left|\nabla T_{k}\left(\left(u_{n}-u_{n+1}\right)^{+}\right)\right|^{2}}{\left(1+T_{n}\left(u_{n}\right)\right)^{p}}\left(1+T_{n}\left(u_{n}\right)\right)^{p} \\
\leq C k^{2} \int_{\left\{0 \leq u_{n}-u_{n+1} \leq k\right\}} \frac{\left|\nabla u_{n+1}\right|^{2}(1+n)^{p}}{\left(1+T_{n+1}\left(u_{n+1}\right)\right)^{p}},
\end{gathered}
$$

$$
\left|\left\{0 \leq u_{n}-u_{n+1} \leq k\right\}\right| \leq C \int_{\left\{0 \leq u_{n}-u_{n+1} \leq k\right\}} \frac{\left|\nabla u_{n+1}\right|^{2}(1+n)^{p}}{\left(1+T_{n+1}\left(u_{n+1}\right)\right)^{p}} .
$$

The right hand side of the above inequality tends to 0 , as $k \rightarrow 0$. Therefore $\mid\left\{0 \leq u_{n}-\right.$ $\left.u_{n+1} \leq k\right\} \mid \rightarrow 0$ as $k \rightarrow 0$. This implies that $u_{n} \leq u_{n+1}$ a.e. in $\Omega$.

We remark that $u_{1}$ is bounded, that is, $\left|u_{1}\right| \leq c$, for some positive constant $c$. Setting $h(s)=\int_{0}^{s} \frac{d t}{\left(1+T_{1}(t)\right)^{p}}$, we have

$$
-\operatorname{div}\left(a(x) \nabla\left(h\left(u_{1}\right)\right)\right)=-\operatorname{div}\left(a(x) \frac{\nabla u_{1}}{\left(1+T_{1}\left(u_{1}\right)\right)^{p}}\right) \geq \frac{T_{1}(f)}{(c+1)^{\gamma}} .
$$

Let $z$ be the $H_{0}^{1}(\Omega)$ solution to $-\operatorname{div}(a(x) \nabla z)=\frac{T_{1}(f)}{(c+1)^{\gamma}}$. By the strong maximum principle, for every $\omega \subset \subset \Omega$ there exists a positive constant $c_{\omega}$ such that $z \geq c_{\omega}$ a.e. in $\omega$. By the comparison principle, we have $h\left(u_{1}\right) \geq z$ a.e. in $\Omega$. The strict monotonicity of $h$ implies the existence of a constant $c_{\omega}>0$, for every $\omega \subset \subset \Omega$, such that $u_{1} \geq c_{\omega}$ a.e. in $\omega$. Since $u_{n}$ is an increasing sequence, as proved above, $u_{n} \geq c_{\omega}$ a.e. in $\omega$ for every $n \in \mathbb{N}$.

\section{Existence RESUlts}

We are going to prove in the following three lemmata some a priori estimates on the solutions $u_{n}$ to problems (2.1). They will allow us to show Theorem 1.1. In the proofs $C$ will denote a positive constant independent of $n$.

Lemma 3.1. Assume that $p-1 \leq \gamma<p+1$.

(1) Let $f \in L^{m}(\Omega)$, with $m \geq \frac{2^{*}}{2^{*}-p-1+\gamma}$. Then the solutions $u_{n}$ to (2.1) are uniformly bounded in $H_{0}^{1}(\Omega)$. If $\frac{2^{*}}{2^{*}-p-1+\gamma} \leq m<\frac{N}{2}$ then the solutions $u_{n}$ are uniformly bounded in $L^{m^{* *}(\gamma+1-p)}(\Omega)$. 
(2) Let $f \in L^{m}(\Omega)$, with $\max \left\{1, \frac{1^{*}}{2 \cdot 1^{*}-p-1+\gamma}\right\}<m<\frac{2^{*}}{2^{*}-p-1+\gamma}$. Then the solutions $u_{n}$ to (2.1) are uniformly bounded in $W_{0}^{1, \sigma}(\Omega), \sigma=\frac{N m(\gamma+1-p)}{N+\gamma m-m(p+1)}$.

Proof. In case (1) let us choose $\left(1+u_{n}\right)^{p+1}-1$ as a test function; the hypotheses on $a$ imply that

$$
\alpha(p+1) \int_{\Omega} \frac{\left|\nabla u_{n}\right|^{2}}{\left(1+T_{n}\left(u_{n}\right)\right)^{p}}\left(1+u_{n}\right)^{p} \leq C \int_{\Omega}|f| u_{n}^{p+1-\gamma} .
$$

By Sobolev's inequality on the left hand side and Hölder's inequality with exponent $\bar{m}=$ $\frac{2^{*}}{2^{*}-p-1+\gamma}=\frac{2 N}{N(\gamma+1-p)+2(p+1-\gamma)}(>1)$ on the right one, we have

$$
\mathcal{S} \alpha(p+1)\left\|u_{n}\right\|_{L^{2^{*}(\Omega)}}^{2} \leq \alpha(p+1)\left\|\nabla u_{n}\right\|_{L^{2}(\Omega)}^{2} \leq\|f\|_{L^{m}(\Omega)}\left[\int_{\Omega}\left|u_{n}\right|^{\bar{m}^{\prime}(p+1-\gamma)}\right]^{\frac{1}{\bar{m}^{\prime}}} .
$$

We remark that $2^{*}=\bar{m}^{\prime}(p+1-\gamma)$. Moreover $\frac{2}{2^{*}} \geq \frac{1}{\bar{m}^{\prime}}$ as $\gamma \leq p+1$. Then the above estimate implies that the sequence $u_{n}$ is bounded in $L^{2^{*}}(\Omega)$ and in $H_{0}^{1}(\Omega)$.

We are now going to prove that $u_{n}$ is bounded in $L^{m^{* *}(\gamma+1-p)}(\Omega)$, if $m<\frac{N}{2}$. Let us choose $\left(1+u_{n}\right)^{\delta}-1$ as a test function: by the hypotheses on $a$, one has

$$
\begin{gathered}
\frac{4 \alpha \delta}{(-p+\delta+1)^{2}} \int_{\Omega}\left|\nabla\left[\left(1+u_{n}\right)^{\frac{-p+\delta+1}{2}}-1\right]\right|^{2}=\alpha \delta \int_{\Omega} \frac{\left|\nabla u_{n}\right|^{2}}{\left.\left(1+u_{n}\right)\right)^{p-\delta+1}} \\
\leq \alpha \delta \int_{\Omega} \frac{\left|\nabla u_{n}\right|^{2}}{\left(1+T_{n}\left(u_{n}\right)\right)^{p}}\left(1+u_{n}\right)^{\delta-1} \leq \int_{\Omega} \frac{T_{n}(f)}{\left(u_{n}+\frac{1}{n}\right)^{\gamma}}\left[\left(u_{n}+1\right)^{\delta}-1\right] \leq C+C \int_{\Omega} \frac{|f|}{\left(u_{n}+1\right)^{-\delta+\gamma}} .
\end{gathered}
$$

By Sobolev's inequality on the left hand side and Hölder's inequality on the right one we have

$$
\left[\left.\int_{\Omega}\left[\left(1+u_{n}\right)^{\frac{-p+\delta+1}{2}}-1\right]\right|^{2^{*}}\right]^{\frac{2}{2^{*}}} \leq C\|f\|_{L^{m}(\Omega)}\left[\int_{\Omega}\left|u_{n}+1\right|^{m^{\prime}(\delta-\gamma)}\right]^{\frac{1}{m^{\prime}}} .
$$

Let $\delta$ be such that $\frac{(1+\delta-p) N}{N-2}=\frac{(\delta-\gamma) m}{m-1}$ and $\frac{2}{2^{*}} \geq \frac{1}{m^{\prime}}$, that is, $\delta=\frac{(1-p) N(m-1)+\gamma m(N-2)}{N-2 m}$ and $m \leq \frac{N}{2}$. We observe that $(-p+\delta+1) \frac{2^{*}}{2}=m^{* *}(\gamma+1-p)>1$. This implies that $u_{n}$ is bounded in $L^{m^{* *}(\gamma+1-p)}(\Omega)$.

In case (2), let us choose $\left(1+u_{n}\right)^{\theta}-1, \theta=\frac{(p-1) N(m-1)-\gamma m(N-2)}{2 m-N}$, as a test function. With the same arguments as before, we have

$$
\left[\left.\int_{\Omega}\left[\left(1+u_{n}\right)^{\frac{-p+\theta+1}{2}}-1\right]\right|^{2^{*}}\right]^{\frac{2}{2^{*}}} \leq C \int_{\Omega} \frac{\left|\nabla u_{n}\right|^{2}}{\left.\left(1+u_{n}\right)\right)^{p-\theta+1}} \leq C\|f\|_{L^{m}(\Omega)}\left[\int_{\Omega}\left|u_{n}+1\right|^{m^{\prime}(\theta-\gamma)}\right]^{\frac{1}{m^{\prime}}} .
$$

As above, we infer that $u_{n}$ is bounded in $L^{\frac{N(1+\theta-p)}{N-2}}(\Omega)$. We observe that $p-\theta+1>0$ and $1<\sigma=\frac{N m(\gamma+1-p)}{N-m(p+1-\gamma)}<2$, by the assumptions on $m$. Writing

$$
\int_{\Omega}\left|\nabla u_{n}\right|^{\sigma}=\int_{\Omega} \frac{\left|\nabla u_{n}\right|^{\sigma}}{\left(1+u_{n}\right)^{\sigma \frac{p-\theta+1}{2}}}\left(1+u_{n}\right)^{\sigma \frac{p-\theta+1}{2}}
$$


and using Hölder's inequality with exponent $\frac{2}{\sigma}$, we obtain

$$
\int_{\Omega}\left|\nabla u_{n}\right|^{\sigma} \leq\left[\int_{\Omega} \frac{\left|\nabla u_{n}\right|^{2}}{\left(1+u_{n}\right)^{p-\theta+1}}\right]^{\frac{\sigma}{2}}\left[\int_{\Omega}\left(1+u_{n}\right)^{\sigma \frac{p-\theta+1}{2-\sigma}}\right]^{\frac{2-\sigma}{2}} .
$$

The above estimates imply that the sequence $u_{n}$ is bounded in $W_{0}^{1, \sigma}(\Omega)$ if $\sigma \frac{p-\theta+1}{2-\sigma}=$ $\frac{N(1+\theta-p)}{N-2}$, that is, $\sigma=\frac{N m(\gamma+1-p)}{N-m(p+1-\gamma)}$.

Lemma 3.2. Assume that $\gamma=p+1$ and $f \in L^{1}(\Omega)$. Then the solutions $u_{n}$ to 2.1) are uniformly bounded in $H_{0}^{1}(\Omega)$.

Proof. Let us choose $\left(1+u_{n}\right)^{p+1}-1$ as a test function. Using that $a(x) \geq \alpha$ a.e. in $\Omega$, we have

$$
\alpha(p+1) \int_{\Omega} \frac{\left|\nabla u_{n}\right|^{2}}{\left(1+T_{n}\left(u_{n}\right)\right)^{p}}\left(1+u_{n}\right)^{p} \leq C \int_{\Omega}|f|
$$

The previous estimate implies that the sequence $u_{n}$ is bounded in $H_{0}^{1}(\Omega)$.

Lemma 3.3. Assume that $\gamma>p+1$ and $f \in L^{1}(\Omega)$. Then the solutions $u_{n}$ to 2.1) are such that $u_{n}^{\frac{\gamma+1-p}{2}}$ is uniformly bounded in $H_{0}^{1}(\Omega), u_{n}$ is uniformly bounded in $L^{\frac{\gamma+1-p}{2} 2^{*}}(\Omega)$ and in $H_{l o c}^{1}(\Omega)$.

Proof. If we choose $u_{n}^{\gamma}$ as a test function and use the hypotheses on $a$ we get

$$
\frac{4 \alpha \gamma}{(\gamma+1-p)^{2}} \int_{\Omega}\left|\nabla\left(u_{n}^{\frac{\gamma+1-p}{2}}\right)\right|^{2}=\alpha \gamma \int_{\Omega}\left|\nabla u_{n}\right|^{2} u_{n}^{\gamma-1-p} \leq \int_{\Omega}|f| .
$$

This proves that the sequence $u_{n}^{\frac{\gamma+1-p}{2}}$ is bounded in $H_{0}^{1}(\Omega)$. Sobolev's inequality on the left hand side applied to $u_{n}^{\frac{\gamma+1-p}{2}}$ gives

$$
\int_{\Omega} u_{n}^{\frac{\gamma+1-p}{2} 2^{*}} \leq C .
$$

Let us prove that $u_{n}$ is bounded in $H_{l o c}^{1}(\Omega)$. For $\varphi \in C_{0}^{1}(\Omega)$ we choose $\left[\left(u_{n}+1\right)^{p+1}-1\right] \varphi^{2}$ as a test function in (2.1). Then, if $\omega=\{\varphi \neq 0\}$, one has by the hypotheses on $a$ and Lemma 2.3

$$
\begin{aligned}
\alpha(p+1) \int_{\Omega}\left|\nabla u_{n}\right|^{2} \varphi^{2} & +2 \alpha \int_{\Omega} \nabla u_{n} \cdot \nabla \varphi \varphi u_{n} \leq \int_{\Omega} \frac{|f|}{\left(u_{n}+\frac{1}{n}\right)^{\gamma}}\left[\left(u_{n}+1\right)^{p+1}-1\right] \varphi^{2} \\
& \leq \int_{\Omega} C_{\omega}|f| \varphi^{2} \leq C_{\omega}\|\varphi\|_{L^{\infty}(\Omega)}^{2} \int_{\Omega}|f|,
\end{aligned}
$$

where $C_{\omega}$ is a positive constant depending only on $\omega \subset \subset \Omega$ and $p$. Then

$$
(p+1) \int_{\Omega}\left|\nabla u_{n}\right|^{2} \varphi^{2} \leq-2 \int_{\Omega} \nabla u_{n} \cdot \nabla \varphi \varphi u_{n}+\frac{C_{\omega}}{\alpha}\|\varphi\|_{L^{\infty}(\Omega)}^{2} \int_{\Omega}|f| .
$$

Young's inequality implies that

$$
2\left|\int_{\Omega} \nabla u_{n} \cdot \nabla \varphi \varphi u_{n}\right| \leq \int_{\Omega}\left|\nabla u_{n}\right|^{2} \varphi^{2}+\int_{\Omega}|\nabla \varphi|^{2} u_{n}^{2} .
$$

From (3.2) we infer that

$$
p \int_{\Omega}\left|\nabla u_{n}\right|^{2} \varphi^{2} \leq \int_{\Omega}|\nabla \varphi|^{2} u_{n}^{2}+\frac{C_{\omega}}{\alpha}\|\varphi\|_{L^{\infty}(\Omega)}^{2} \int_{\Omega}|f| .
$$


Since $u_{n}$ is uniformly bounded in $L^{2}(\Omega)$ by (3.1), this proves our result.

We are now able to prove Theorem 1.1

Proof. We will prove point (1); the second and the third point can be proved in a similar way. Lemma 3.1 gives the existence of a function $u \in H_{0}^{1}(\Omega)$ such that $u_{n} \rightarrow u$ weakly in $H_{0}^{1}(\Omega)$ and a.e. in $\Omega$, up to a subsequence. We will prove that $u$ is a solution to (1.1) passing to the limit in (2.1). For every $\varphi \in C_{0}^{1}(\Omega)$,

$$
\int_{\Omega} \frac{a(x) \nabla u_{n} \cdot \nabla \varphi}{\left(1+T_{n}\left(u_{n}\right)\right)^{p}} \rightarrow \int_{\Omega} \frac{a(x) \nabla u \cdot \nabla \varphi}{(1+u)^{p}}
$$

since $\frac{1}{\left(1+T_{n}\left(u_{n}\right)\right)^{p}} \rightarrow \frac{1}{(1+u)^{p}}$ in $L^{r}(\Omega)$, for every $r \geq 1$. For the limit of the right hand side of (2.1), let $\omega=\{\varphi \neq 0\}$. One can use Lebesgue's theorem, since

$$
\left|\frac{T_{n}(f) \varphi}{\left(u_{n}+\frac{1}{n}\right)^{\gamma}}\right| \leq \frac{|\varphi||f|}{c_{\omega}^{\gamma}}
$$

where $c_{\omega}$ is the constant given by Lemma 2.3. In the case where $\frac{2^{*}}{2^{*}-p-1+\gamma} \leq m<\frac{N}{2}$, since $m^{* *}(\gamma+1-p)>1$, Lemma 3.1 implies that $u_{n}$ converges weakly in $L^{m^{* *}(\gamma+1-p)}(\Omega)$ to some function, which is $u$ by identification.

To prove that $u$ is bounded for $\gamma \geq p-1$, let us choose $\left[\left(u_{n}+1\right)^{\gamma+1}-(k+1)^{\gamma+1}\right]_{+}$as a test function in (2.1):

$$
\alpha(\gamma+1) \int_{A_{k}} \frac{\left|\nabla u_{n}\right|^{2}}{\left(1+u_{n}\right)^{p-\gamma}} \leq \int_{A_{k}}|f| \frac{\left(u_{n}+1\right)^{\gamma+1}-(k+1)^{\gamma+1}}{\left(u_{n}+\frac{1}{n}\right)^{\gamma}} \leq c(\gamma) \int_{A_{k}}|f|\left(u_{n}-k\right),
$$

where $A_{k}=\left\{u_{n} \geq k\right\}$ and $c(\gamma)$ denotes a positive constant depending only on $\gamma$.

For $p-\gamma \leq 0$, (3.3) is the starting point of of the proof of Theorem 4.1 in [14]. For $0<p-\gamma \leq 1$, (3.3) is the starting point of Lemma 2.2 in [7. In both cases $u_{n}$ is uniformly bounded in $L^{\infty}(\Omega)$ and therefore (at the a.e. limit) the solutions $u$ found in the previous results are bounded.

REMARK 3.4. We observe that we have the boundedness of the solution to problem (1.1) for any value of $\gamma \geq p-1$.

\section{ACKNOWLEDGMENTS}

Part of this work was done during a visit to La Sapienza Università di Roma whose hospitality is gratefully acknowledged.

\section{REFERENCES}

[1] A. Alvino, Angelo, L. Boccardo, V. Ferone, L. Orsina and G. Trombetti, Existence results for nonlinear elliptic equations with degenerate coercivity, Ann. Mat. Pura Appl. 4 (2003), no. 1, 5379

[2] D. Arcoya, J. Carmona, T. Leonori, P.J. Martínez-Aparicio, L. Orsina and F. Petitta, Existence and non-existence of solutions for singular quadratic quasilinear equations, J. Differential Equations 246 (2009), 4006-4042.

[3] L. Boccardo, On the regularizing effect of strongly increasing lower order terms, J. Evol. Equ. 3 (2003), 225-236.

[4] L. Boccardo, Quasilinear elliptic equations with natural growth terms: the regularizing effects of the lower order terms, J. Nonlin. Conv. Anal. 7 (2006), 355-365.

[5] L. Boccardo, Dirichlet problems with singular and gradient quadratic lower order terms, ESAIM Control Optim. Calc. Var. 14 (2008), 411-426. 
[6] L. Boccardo and H. Brezis, Some remarks on a class of elliptic equations with degenerate coercivity, Boll. Unione Mat. Ital. 6 (2003), 521-530.

[7] L. Boccardo, A. Dall'Aglio and L. Orsina, Existence and regularity results for some elliptic equations with degenerate coercivity. Dedicated to Prof. C. Vinti (Italian) (Perugia, 1996), Atti Sem. Mat. Fis. Univ. Modena 46 suppl. no. 5 (1998), 1-81.

[8] L. Boccardo and L. Orsina, Semilinear elliptic equations with singular nonlinearities, Calc. Var. Partial Differential Equations 37 (2010), 363-380.

[9] H. Brézis and W. Strauss, Semi-linear second-order elliptic equations in $L^{1}$, J. Math. Soc. Japan 25 (1973), 565-590.

[10] G. Croce, The regularizing effects of some lower order terms on the solutions in an elliptic equation with degenerate coercivity, Rendiconti di Matematica Serie VII 27 (2007), 200-314.

[11] G. Croce, An elliptic problem with degenerate coercivity and a singular quadratic gradient lower order term, to appear in Discr. Contin. Dyn. Syst. - S.

[12] J. Leray and J.-L. Lions, Quelques résulatats de Višik sur les problèmes elliptiques nonlinéaires par les méthodes de Minty-Browder, Bull. Soc. Math. France 93 (1965), 97-107.

[13] A. Porretta, Uniqueness and homogeneization for a class of noncoercive operators in divergence form, Atti Sem. Mat. Fis. Univ. Modena 46 suppl. (1998), 915-936.

[14] G. Stampacchia, Le problème de Dirichlet pour les équations elliptiques du second ordre à coefficients discontinus, Ann. Inst. Fourier (Grenoble) 15 (1965), 189-258.

Laboratoire de Mathématiques Appliquées du Havre, Université du Havre, 25, rue Philippe LEBon, 76063 Le Havre (FRANCE)

E-mail address: gisella.croce@univ-lehavre.fr 